\title{
Effect of Foliar Spray of Humic acid, Ascorbic acid, Cultivars and their Interactions on Growth of Olive (Olea European L.) Transplants cvs. Khithairy and Sorany
}

\author{
Azad Ahmed Mayi, Zulaikha Ramazan Ibrahim and Amira Salih Abdurrahman \\ Horticulture Department, School of plant production, Faculty of Agriculture and forestry, Duhok University, \\ Kurdistan Region, Iraq
}

\begin{abstract}
The study was conducted at the nursery of Malta station Duhok, Kurdistan region - Iraq, during the season 2011 to study the effect of ascorbic acid and humic acid on vegetative growth of two olive (Olea europaea) cultivars (Khithairy and Sorany). The transplants were sprayed with four levels (0, 500, 1000 and $\left.2000 \mathrm{mg} . \mathrm{l}^{-1}\right)$ ascorbic acid and four levels of humic acid $\left(0,20,40\right.$ and $\left.60 \mathrm{mg} . \mathrm{l}^{-1}\right)$. When transplant treated with $500 \mathrm{mg} . \mathrm{l}^{-1}$ ascorbic acid showed significantly effect of most characteristics, however foliar application of humic acid at $20 \mathrm{mg} . \mathrm{l}^{-1}$ significantly increase plant height, leaf fresh weight and leaf dry weight. Khithairy cultivar significantly dominated Sorany cultivar of the most studied characteristics. The interactions between cultivar and ascorbic acid showed significantly affected on the most characteristic studied, while Khithairy cultivar when treated with $500 \mathrm{mg} . \mathrm{l}^{-1}$ ascorbic acid significantly increased plant height, leaves number, leaf area and lateral shoot number. Cultivar and humic acid interactions showed that the Khithairy when treated with 60 $m g . l^{-1}$ humic acid significantly affected on the most of the characteristic studied while the interactions between ascorbic acid and humic showed that the transplants when treated with 20mg..$^{-1}$ humic only increase significantly most characteristics. The interactions between (khithairy $\times 0 \mathrm{mg} . \mathrm{l}^{-1}$ ascorbic acid $\times 60 \mathrm{mg} . l^{-1}$ humic) increased significantly leaves number, shoot length and lateral shoot number.
\end{abstract}

Key word: Humic acid, Ascorbic acid, Olive cultivar, leaf nutrient.

\section{Introduction}

Olive belongs to the botanical order, Ligustrales, family Oleaceae; this family includes 30 genus including Olea and has 600 species. Olive is botanically called (Olea europaea L.). Commercial olives belong to the Europaea species, this species has two subspecies: oleaster and sativa [1] and [2].

World olive production performs an important role in the economy of many countries such as Spain, Italy, Greece, Turkey and Tunisia. Olive is an important perennial crop in many agricultural regions of the Mediterranean countries, as it is the most important olive growing region. The olive tree yield has two main products: oil and table olives.

In Iraq, olive trees growing in some areas of central and Kurdistan of Iraq, Nineveh is the governorate leading olive producer, its cultivation in Nineveh spreading in an area including village of Baashiqa, Bahzany, Fadiliya, Sheikh Uday, Dhecan, Sinjar, Aqrah, Bamarni followed by Babylon, Diyala, Kirkuk, Baghdad, Erbil and Duhok [2] and[3].

Organic fertilizers as well as the remains of animal waste are also a kind of humus, which contribute the soil fertility [4]. Organic fertilizers are natural materials and a good medium for the interaction of micro-organisms and provide plant with nutrients as well as having an indirect role in nutrition by the activity of microorganisms. So using organic and bio-fertilizers instead of the chemical forms could be the way to produce the natural healthy fruits. In this respect, the organic fertilization improved vegetative growth, nutritional status [5].

The application of organic fertilizers avoided pollution and reduced the cost of fertilization. Also, it has drowned the attention of olive growers to use the organic and bio- fertilizers that would be healthy for human and safe for the environment [6].

Humic acids (HAs) are the main fractions of humic substances (HS) and the most active components of soil and compost organic matter. HAs have been shown to stimulate plant growth and consequently yield by acting on mechanisms involved in: cell respiration, photosynthesis, protein synthesis, water and nutrient uptake, enzyme activities [7] and [8].This action of HAs has been demonstrated to be dose dependent and particularly effective in a low concentration range [9]. In particular, optimal concentrations able to affect and stimulate plant growth have been generally found in the range of $50-300 \mathrm{mg} \mathrm{L}^{-1}$, but positive effects have been also exerted by lower concentrations [8].

A distinction on the effects of HAs should be made between indirect and direct effects on plants growth. Indirect effects are mainly exerted through properties such as: enrichment in soil nutrients, increase of microbial population, higher cation exchange capacity (CEC), improvement of soil structure; whereas direct 
effects are various biochemical actions exerted at the cell wall, membrane or cytoplasm and mainly of hormonal nature [10]. The hormone like activities of HAs is well documented in various papers, in particular auxin-, cytokinin- and gibberellins like effects [11], [12] and [13].

Ascorbic acid (Vitamin C) acts as coenzyme reaction shrub native to South Sea Island and the Malaya by which carbohydrates, fats and protein and Peninsula. House plant specimens are pot grown to a metabolized. Vitamin C led to increase nucleic acid content single stem, up to 2 feet high, with smooth leathery leaves especially RNA.

Nowadays, there is a widespread use of antioxidants especially ascorbic acid as natural and organic antioxidant compound. Ascorbic acid is an essential compound for plant tissues since it has antioxidant functions, and acts as co-enzyme in an enzymatic cofactor and plant growth regulator [14]. It has synergistic effect on improving growth, flowering, yield and fruit quality of fruit crops [15], [16], [17] and [18].

[19] reported that marked in vivid coloures. The plants are exacting in their ascorbic acid is an abundant component of plans. It requirements, ample light, high humidity, an even reaches a concentration of over $20 \mathrm{mM}$ in chloroplasts temperature and watering and syringing in summer, less and occurs in all cell compartments including cell wall. It in winter conjugates with sugars and are metabolized by oxidation indicated that application of ascorbic acid significantly [20].

[21] found that small antioxidant molecule vitamin C (L-ascorbic acid, AA) fulfils essential metabolic functions in the life of plants. They added that AA serves as a co-factor for many enzymes and it contributes to the detoxification of reactive oxygen species (ROS) ([22]. This antioxidant activity of AA is associated with resistance to oxidative stress and longevity in plants. Furthermore, the endogenous level of AA has recently been suggested to be important in the regulation of developmental senescence and plant defense against oxidative stress. Plants synthesize AA via several distinct pathways including routes via L-galactose and gulose[22]. Due to the fact that AA also serves as an important co-factor in the biosynthesis of many plant hormones, including ethylene, gibberellic acid, and abscisic acid, one has to assume that the endogenous level of AA will affect not only the biosynthesis, but also the levels and therefore the signaling of these phytohormones. This investigation aimed to Study the effect of humic acid and ascorbic acid on vegetative parameters of Khithairy and Sorany in the climate at Kurdistan region, Find out a fertilization program can replace the mineral which will be beneficial for organic production of olives and save human health and environment.

\section{Materials and Methods}

The study was carried out during 2011 in the nursery of Malta station/ Duhok. Kurdistan region- Iraq. Uniform and healthy olive cvs. Khithairy and Sorany transplant of (2) years old. The experiments were started in April 17th 2011, as transplants were grown in pots each of $(5 \mathrm{~kg})$ weight, filled with river loamy soil [23], four humic acid (HA) concentrations (0, 20,40 and 60.mg. $\mathrm{l}^{-1}$ ), four Ascorbic acid (AA)concentrations (0, 500,1000 and $\left.2000 \mathrm{mg} . \mathrm{l}^{-1}\right)$, and their interactions were sprayed at 17th April and repeated at same concentrations in 17th May [24].

Soil

Taken $(5 \mathrm{~kg}$ ) from soil studied and analyzed which used in the experiment, they were air dried, grounded and passed through a $2 \mathrm{~mm}$ sieve, to prepare them for soil physical and chemical analysis according to [Page and etal,1982 and Official methods of analysis1995]. Methods at soil department laboratory, Agriculture College, Duhok University (Table 1).

The transplants were foliar sprayed with solutions containing humic acid and Ascorbic acid to drip point (run off) using 2 litters hand sprayer at $17^{\text {th }}$ April, 2011. The transplants were left under nursery conditions till end of the experiments. The experiment was arranged as Factorial Randomized Complete Block Design.

Table (1): Some physical and chemical properties of nursery soil.

\begin{tabular}{|l|c|}
\hline Soil properties & Value \\
\hline PH & 7.1 \\
\hline Total nitrogen N\% & 0.07 \\
\hline Available Phosphorus (ppm) & 5.2 \\
\hline${\text { Exchangeable } \mathbf{K}^{+} \text {meq/L }}^{\text {L }}$ & $\mathbf{0 . 0 3 3}$ \\
\hline Exchangeable $\mathbf{C a}^{+2}$ meq/L & 1.2 \\
\hline CaCo3 meq/L & 12.5 \\
\hline EC (ds.m) & 0.20 \\
\hline Organic matter $(\%)$ & 0.34 \\
\hline Sand\% & 62.05 \\
\hline Silt\% & 22.99 \\
\hline Clay\% & 14.96 \\
\hline Soil texture & Loamy sand \\
\hline
\end{tabular}

(R.C.B.D) using three factors $(2 \times 4 \times 4)$ cultivars, humic acid and Ascorbic acid levels consequently, with three replicate. Each replicate consisted of five transplants. The results were analyzed statistically, and comparisons 
were made using Duncan's multiple range tests at 5\% probability [25]. All the data were tabulated and statistically analyzed with computer using [26].

\section{The following measurements were recorded on $15^{\text {th }}$ September 2012.}

1- Plant height $(\mathrm{cm})$ : Length of the main stem of each transplant was measured by using the metric ruler.

2-Stem diameter $(\mathrm{mm})$ was measured digital Varner at $5 \mathrm{~cm}$ above soil surface.

3-Leaf numbers/ plant

4-Singel leaf area $\left(\mathrm{cm}^{2}\right)$

5- Lateral shoots length average $(\mathrm{cm})$. On each experimental unit; 10curent season shoots were selected randomly and labeled at the beginning of the experiment. The increase in shoot length was estimated on $15^{\text {th }}$ September 2011 [27].

6- Lateral shoots number/ plant. Lengths per transplant $(\mathrm{cm})$ were measured by using the metric ruler was obtained on the average by this equation.

7- Total Chlorophyll Content (\%) It was determined by using a chlorophyll measurement device (Chlorophyll meter, SPAD- 502, Konica Minolta). The estimated chlorophyll in leaves represent its ratio to the rest of pigments present in the leaves.

8- Leaf fresh weight (mg).

9- Leaf dry weight $(\mathrm{mg})$. For each randomly transplant 50 leaves were taken. These leaves were cleaned and washed several times with tap water, then washed again by $\mathrm{HCl} 0.01 \mathrm{~N}$ and rinsed by distilled water to remove any spray residues. After the leaves weight was taken, they were oven-dried at $70^{\circ} \mathrm{C}$ until constant weight [28].

\subsection{Plant high (cm):}

\section{Results:}

The obtained results of (Table 2) revealed that spraying olive transplants with humic acid concentrations resulted in a significant increase in plant high, particularly at $63 \mathrm{mg} .1^{-1}$ level as compared to the control.

Table (2) Effect of humic acid, ascorbic acid, cultivar and their interactions on plant high (cm) of olive transplant cvs. 'Khithairy ' and ' Sorany'

\begin{tabular}{|c|c|c|c|c|c|c|c|}
\hline \multirow{2}{*}{$\stackrel{\vec{E}}{\stackrel{\vec{E}}{E}}$} & \multirow{2}{*}{ 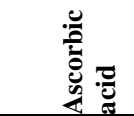 } & \multicolumn{4}{|c|}{ Humic concentration } & \multirow[b]{2}{*}{$\begin{array}{l}\text { cultivar } \times \text { ascorbic } \\
\text { acid }\end{array}$} & \multirow[b]{2}{*}{$\begin{array}{l}\text { cultivar } \\
\text { effect }\end{array}$} \\
\hline & & $\mathbf{0}$ & 20 & 40 & 60 & & \\
\hline \multirow{4}{*}{ 昰 } & $\mathbf{0}$ & $\begin{array}{l}39.67 \\
q\end{array}$ & $\begin{array}{l}71.67 \\
b-e\end{array}$ & $\begin{array}{l}73.33 \\
\text { a-c }\end{array}$ & $\begin{array}{l}69.67 \\
c-f\end{array}$ & $\begin{array}{l}63.58 \\
d\end{array}$ & \multirow{4}{*}{69.08} \\
\hline & 500 & $\begin{array}{l}76.33 \\
\mathrm{a}\end{array}$ & $\begin{array}{l}73.67 \\
a b\end{array}$ & $\begin{array}{l}74.67 \\
a b\end{array}$ & $\begin{array}{l}73 \\
\text { a-d }\end{array}$ & $\begin{array}{l}74.42 \\
\mathrm{a} \\
\end{array}$ & \\
\hline & 1000 & $\begin{array}{l}68.67 \\
\text { e-g }\end{array}$ & $\begin{array}{l}72.33 \\
b-e\end{array}$ & $\begin{array}{l}71.33 \\
\text { b-f }\end{array}$ & $\begin{array}{l}69.33 \\
\mathrm{~d}-\mathrm{g}\end{array}$ & $\begin{array}{l}70.42 \\
b\end{array}$ & \\
\hline & 2000 & $\begin{array}{l}65.67 \\
\text { gh }\end{array}$ & $\begin{array}{l}68.67 \\
\text { e-g }\end{array}$ & $\begin{array}{l}69.67 \\
c-f\end{array}$ & $\begin{array}{l}67.67 \\
\text { fg }\end{array}$ & $\begin{array}{l}67.92 \\
\mathrm{c}\end{array}$ & \\
\hline \multirow[b]{4}{*}{ 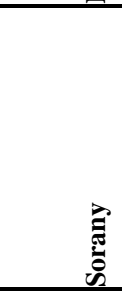 } & $\mathbf{0}$ & $\begin{array}{l}42.33 \\
\mathrm{pq}\end{array}$ & $\begin{array}{l}63 \\
\mathrm{~h}\end{array}$ & $\begin{array}{l}56.67 \\
\mathrm{ij}\end{array}$ & $\begin{array}{l}48 \\
\mathrm{mn}\end{array}$ & $\begin{array}{l}52.5 \\
\mathrm{e} \\
\end{array}$ & \multirow[t]{4}{*}{$\begin{array}{l}51.94 \\
\mathrm{~b}\end{array}$} \\
\hline & 500 & $\begin{array}{l}53 \\
j-1\end{array}$ & $\begin{array}{l}57 \\
\mathrm{i}\end{array}$ & $\begin{array}{l}55.67 \\
\text { ij }\end{array}$ & $\begin{array}{l}50.67 \\
k-m\end{array}$ & $\begin{array}{l}54.08 \\
\mathrm{e}\end{array}$ & \\
\hline & 1000 & $\begin{array}{l}44 \\
\text { op }\end{array}$ & $\begin{array}{l}53.67 \\
\mathrm{i}-\mathrm{k}\end{array}$ & $\begin{array}{l}50.33 \\
k-m\end{array}$ & $\begin{array}{l}46.33 \\
\text { no }\end{array}$ & $\begin{array}{l}48.58 \\
\mathrm{f}\end{array}$ & \\
\hline & 2000 & $\begin{array}{l}50.33 \\
k-m\end{array}$ & $\begin{array}{l}57 \\
\mathrm{i}\end{array}$ & $\begin{array}{l}53.67 \\
\text { i-k }\end{array}$ & $\begin{array}{l}49.33 \\
1-n\end{array}$ & $\begin{array}{l}52.58 \\
\mathrm{e}\end{array}$ & \\
\hline \multicolumn{2}{|c|}{ Humic effect } & & $\begin{array}{l}64.63 \\
\mathrm{a}\end{array}$ & $\begin{array}{l}63.17 \\
b\end{array}$ & $\begin{array}{l}59.25 \\
\mathrm{c}\end{array}$ & \multirow[b]{3}{*}{$\begin{array}{c}\text { Ascorbic acid } \\
\text { effect }\end{array}$} & \\
\hline \multirow{2}{*}{ 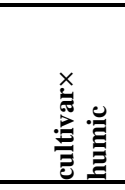 } & Khithairy & $\begin{array}{l}62.58 \\
\mathrm{c} \\
\end{array}$ & $\begin{array}{l}71.58 \\
\mathrm{ab} \\
\end{array}$ & $\begin{array}{l}72.25 \\
\mathrm{a} \\
\end{array}$ & $\begin{array}{l}69.92 \\
\mathrm{~b} \\
\end{array}$ & & \\
\hline & Sorany & $\begin{array}{l}47.42 \\
\mathrm{f} \\
\end{array}$ & $\begin{array}{l}57.67 \\
\mathrm{~d}\end{array}$ & $\begin{array}{l}54.08 \\
\mathrm{e} \\
\end{array}$ & $\begin{array}{l}48.58 \\
\mathrm{f} \\
\end{array}$ & & \\
\hline \multirow{4}{*}{ 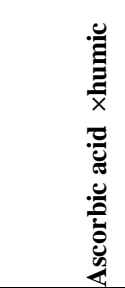 } & $\mathbf{0}$ & $\begin{array}{l}41 \\
\mathrm{f}\end{array}$ & $\begin{array}{l}67.33 \\
\mathrm{a} \\
\end{array}$ & $\begin{array}{l}65 \\
a b\end{array}$ & $\begin{array}{l}58.83 \\
\text { de }\end{array}$ & $\begin{array}{l}58.04 \\
\mathrm{c} \\
\end{array}$ & \\
\hline & 500 & $\begin{array}{l}64.67 \\
\mathrm{Ab}\end{array}$ & $\begin{array}{l}65.33 \\
a b\end{array}$ & $\begin{array}{l}65.16 \\
a b\end{array}$ & $\begin{array}{l}61.83 \\
\mathrm{c}\end{array}$ & $\begin{array}{l}64.25 \\
\mathrm{a}\end{array}$ & \\
\hline & 1000 & $\begin{array}{l}56.33 \\
\mathrm{E}\end{array}$ & $\begin{array}{l}63 \\
b c\end{array}$ & $\begin{array}{l}60.83 \\
\mathrm{~cd}\end{array}$ & $\begin{array}{l}57.83 \\
\mathrm{e}\end{array}$ & $\begin{array}{l}59.5 \\
b\end{array}$ & \\
\hline & 2000 & $\begin{array}{l}58 \\
E\end{array}$ & $\begin{array}{l}62.83 \\
\mathrm{bc}\end{array}$ & $\begin{array}{l}61.66 \\
c\end{array}$ & $\begin{array}{l}58.5 \\
\text { de }\end{array}$ & $\begin{array}{l}60.25 \\
b\end{array}$ & \\
\hline
\end{tabular}


means within a column, row and their interactions followed with the same letters are not significantly different from each other's according to Duncans multiple ranges test at $5 \%$ level.

Olive transplants treated with most of ascorbic acid concentrations substantially increased plant high, especially at 500mg. $1^{-1}$ as compared to those of the control. Results of cultivars revealed that 'Khithairy gave the highest plant high $(69.08 \mathrm{~cm})$ compared with ' Sorany' olive cultivar $(51.49 \mathrm{~cm})$. Results indicated that the combination between ascorbic acid and humic concentrations displayed that $0 \mathrm{mg} .1^{-1}$ ascorbic acid and $20 \mathrm{mg} .1^{-1}$ humic appeared to be the most potent treatment, as it gave the highest plant high $(67.33 \mathrm{~cm})$. Results of cultivars and ascorbic acid concentrations interaction revealed that spraying 'Khithairy ascorbic acid at a rate of 500mg. $1^{-1}$ gave the highest plant high $(74.42 \mathrm{~cm})$. However, the lowest plant high was observed with ' Sorany' olive cultivar $(48.58 \mathrm{~cm})$ when treated by $1000 \mathrm{mg} .1^{-1}$ ascorbic acid.

Results of humic concentrations and cultivars interaction revealed that spraying 'Khithairy 'olive cultivar with humic acid at a rate of $40 \mathrm{mg} .1^{-1}$ resulted in the highest plant high $(72.25 \mathrm{~cm})$. However, untreated 'Sorany' olive cultivar gave the lowest values $(47.42 \mathrm{~cm})$. Results of ascorbic acid, humic and cultivars interaction indicated that spraying 'Khithairy 'olive cultivar with $500 \mathrm{mg} .1^{-1}$ ascorbic acid plus $0 \mathrm{mg} .1^{-1}$ humic was the most potent treatment which gave $76.33 \mathrm{~cm}$ plant high while the lowest plant high coincided with untreated 'Khithairy olive cultivar $(39.67 \mathrm{~cm})$.

\subsection{Stem diameter $(\mathbf{m m})$}

Table (3) showed that the olive transplants were spraying with humic acid concentration $20 \mathrm{mg} . \mathrm{l}^{-1}$ gave the highest stem diameter $(6.96 \mathrm{~mm})$ when compared with the control. The olive transplants treated with ascorbic acid concentration in $1000 \mathrm{mg} .1-1$ the highest value when compared with other concentration.

Table (3) Effect of humic, ascorbic acid , cultivar and their interactions on stem diameter (mm) of olive transplant cvs. 'Khithairy ' and ' Sorany'.

\begin{tabular}{|c|c|c|c|c|c|c|c|}
\hline \multirow{2}{*}{$\stackrel{\Xi}{\Xi}$} & \multirow{2}{*}{ 递 } & \multicolumn{4}{|c|}{ Humic concentration } & \multirow{2}{*}{$\begin{array}{c}\text { cultivar } \\
\times \text { ascorbic acid }\end{array}$} & \multirow{2}{*}{ cultivar effect } \\
\hline & & $\mathbf{0}$ & 20 & 40 & 60 & & \\
\hline \multirow{4}{*}{ 芯 } & $\mathbf{0}$ & $\begin{array}{c}4.33 \\
\mathrm{~h}\end{array}$ & $\begin{array}{c}6.67 \\
\text { b-e }\end{array}$ & $\begin{array}{c}7.33 \\
\mathrm{bc}\end{array}$ & $\begin{array}{c}7.67 \\
\mathrm{a}\end{array}$ & $\begin{array}{c}6.50 \\
\mathrm{ab}\end{array}$ & \multirow{4}{*}{$\begin{array}{c}6.33 \\
\mathbf{a}\end{array}$} \\
\hline & 500 & $\begin{array}{c}6.00 \\
\mathrm{~d}-\mathrm{g}\end{array}$ & $\begin{array}{c}6.33 \\
\mathrm{c}-\mathrm{f}\end{array}$ & $\begin{array}{c}6.67 \\
b-e\end{array}$ & $\begin{array}{l}7.00 \\
b-d\end{array}$ & $\begin{array}{l}6.50 \\
\mathrm{ab}\end{array}$ & \\
\hline & 1000 & $\begin{array}{c}6.00 \\
\mathrm{~d}-\mathrm{g} \\
\end{array}$ & $\begin{array}{c}6.00 \\
\mathrm{~d}-\mathrm{g}\end{array}$ & $\begin{array}{c}6.67 \\
\mathrm{~b}-\mathrm{e}\end{array}$ & $\begin{array}{c}6.33 \\
\mathrm{c}-\mathrm{f}\end{array}$ & $\begin{array}{c}6.25 \\
\mathrm{ab}\end{array}$ & \\
\hline & 2000 & $\begin{array}{c}5.00 \\
\mathrm{gh}\end{array}$ & $\begin{array}{c}6.00 \\
d-g\end{array}$ & $\begin{array}{l}7.00 \\
b-d\end{array}$ & $\begin{array}{c}6.33 \\
\text { c-f }\end{array}$ & $\begin{array}{c}6.08 \\
\mathrm{bc}\end{array}$ & \\
\hline \multirow{4}{*}{ 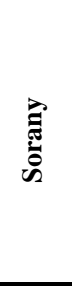 } & $\mathbf{0}$ & $\begin{array}{c}5.67 \\
\mathrm{e}-\mathrm{g}\end{array}$ & $\begin{array}{c}8.67 \\
\mathrm{a}\end{array}$ & $\begin{array}{c}7.33 \\
\mathrm{bc}\end{array}$ & $\begin{array}{c}5.00 \\
\mathrm{gh}\end{array}$ & $\begin{array}{c}6.67 \\
\mathrm{a}\end{array}$ & \multirow{4}{*}{$\begin{array}{c}6.44 \\
a\end{array}$} \\
\hline & 500 & $\begin{array}{c}6.67 \\
\mathrm{~b}-\mathrm{e}\end{array}$ & $\begin{array}{c}7.67 \\
\mathrm{~b}\end{array}$ & $\begin{array}{c}6.67 \\
\mathrm{~b}-\mathrm{e}\end{array}$ & $\begin{array}{c}5.67 \\
\mathrm{e}-\mathrm{g}\end{array}$ & $\begin{array}{c}6.67 \\
\mathrm{a} \\
\end{array}$ & \\
\hline & 1000 & $\begin{array}{c}6.67 \\
\mathrm{~b}-\mathrm{e}\end{array}$ & $\begin{array}{c}7.67 \\
b\end{array}$ & $\begin{array}{c}6.67 \\
\mathrm{~b}-\mathrm{e}\end{array}$ & $\begin{array}{l}5.67 \\
\mathrm{e}-\mathrm{g}\end{array}$ & $\begin{array}{c}6.67 \\
\mathrm{a} \\
\end{array}$ & \\
\hline & 2000 & $\begin{array}{l}5.33 \\
\mathrm{f}-\mathrm{h}\end{array}$ & $\begin{array}{c}6.67 \\
b-e\end{array}$ & $\begin{array}{c}6.00 \\
\text { d-g }\end{array}$ & $\begin{array}{c}5.00 \\
\mathrm{gh}\end{array}$ & $\begin{array}{c}5.75 \\
\mathrm{c} \\
\end{array}$ & \\
\hline \multicolumn{2}{|c|}{ Humic effect } & $\begin{array}{c}5.71 \\
\mathrm{c}\end{array}$ & $\begin{array}{c}6.96 \\
\mathrm{a}\end{array}$ & $\begin{array}{c}6.79 \\
\mathrm{a}\end{array}$ & $\begin{array}{c}6.08 \\
\mathrm{~b}\end{array}$ & \multirow{3}{*}{$\begin{array}{c}\text { Ascorbic acid } \\
\text { effect }\end{array}$} & \\
\hline \multirow{2}{*}{ 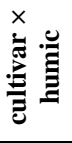 } & Khithairy & $\begin{array}{c}5.33 \\
\mathrm{e}\end{array}$ & $\begin{array}{c}6.25 \\
\mathrm{~cd}\end{array}$ & $\begin{array}{c}6.92 \\
\mathrm{~b}\end{array}$ & $\begin{array}{c}6.83 \\
b\end{array}$ & & \\
\hline & Sorany & $\begin{array}{c}6.08 \\
\mathrm{~d}\end{array}$ & $\begin{array}{c}7.67 \\
\mathrm{a} \\
\end{array}$ & $\begin{array}{c}6.67 \\
\text { bc }\end{array}$ & $\begin{array}{c}5.33 \\
\mathrm{e}\end{array}$ & & \\
\hline \multirow{4}{*}{ 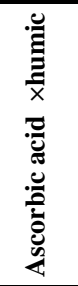 } & $\mathbf{0}$ & $\begin{array}{c}5.00 \\
\mathrm{f}\end{array}$ & $\begin{array}{c}7.66 \\
\mathrm{a} \\
\end{array}$ & $\begin{array}{c}7.33 \\
\mathrm{ab}\end{array}$ & $\begin{array}{c}6.33 \\
\mathrm{c}-\mathrm{e}\end{array}$ & $\begin{array}{c}6.58 \\
\mathrm{a} \\
\end{array}$ & \\
\hline & 500 & $\begin{array}{c}6.33 \\
c-e\end{array}$ & $\begin{array}{l}7.00 \\
a-c\end{array}$ & $\begin{array}{c}6.66 \\
\text { bd }\end{array}$ & $\begin{array}{c}6.33 \\
c-e\end{array}$ & $\begin{array}{c}6.58 \\
\mathrm{a}\end{array}$ & \\
\hline & 1000 & $\begin{array}{c}6.33 \\
c-e\end{array}$ & $\begin{array}{c}6.83 \\
\mathrm{bc}\end{array}$ & $\begin{array}{c}6.66 \\
\text { bd }\end{array}$ & $\begin{array}{c}6.00 \\
\text { de }\end{array}$ & $\begin{array}{c}6.46 \\
\mathrm{a}\end{array}$ & \\
\hline & 2000 & $\begin{array}{c}5.17 \\
\mathrm{f} \\
\end{array}$ & $\begin{array}{c}6.33 \\
\mathrm{c}-\mathrm{e}\end{array}$ & $\begin{array}{c}6.50 \\
\mathrm{~cd}\end{array}$ & $\begin{array}{c}5.66 \\
\text { ef }\end{array}$ & $\begin{array}{c}5.92 \\
\mathrm{~b} \\
\end{array}$ & \\
\hline
\end{tabular}

Means within a column, row and their interactions followed with the same letters are not significantly different from each other's according to Duncan's multiple ranges test at $5 \%$ level.

Results of cultivars revealed that Sorany gave the highest value $(6.44 \mathrm{~mm})$ when compared with Khithairy cultivar (6). Results indicated that the combination between humic acid and ascorbic acid concentration 
displayed that $0 \mathrm{mg} \cdot 1^{-1}$ ascorbic acid and $20 \mathrm{mg} \cdot 1^{-1}$ humic acid are appeared to be the most potent treatment, as it gave the highest stem diameter value $(7.66 \mathrm{~mm})$.

Results of ascorbic acid concentration and cultivar interaction revealed that spraying Sorany with ascorbic acid levels had no significant effect on stem diameter value $(7.25 \mathrm{~mm})$, however the lowest stem diameter was observed with untreated Sorany olive cultivar $(4.75 \mathrm{~mm})$. Result of interaction between Fe concentration and cultivar revealed that spraying Khithairy cultivar with $\mathrm{Fe}$ at a rate $75 \mathrm{mg} . \mathrm{l}^{-1}$ resulted in the highest stem diameter value $(7.58 \mathrm{~mm})$, however Sorany olive cultivar gave the lowest value $(5 \mathrm{~mm})$

The interaction between $\mathrm{GA}_{3}, \mathrm{Fe}$ and Cultivar, when the Khithairy cultivar when treated with $75 \mathrm{mg} . \mathrm{Fe} . \mathrm{I}^{-1}$ plus $0 \mathrm{mg}$. $\mathrm{GA}_{3} \cdot \mathrm{I}^{-1}$ with Sorany cultivar and treated with $0 \mathrm{mg} \cdot \mathrm{Fe} . \mathrm{I}^{-1}$ plus $750 \mathrm{mg} . \mathrm{GA}_{3} \cdot \mathrm{I}^{-1}$ gave the highest value.

\subsection{Leaf number:}

In the table (4) showed foliar application of humic acid increased leaf number, the highest leaf number value (148.00) produced by transplant treated with $60 \mathrm{mg} \cdot \mathrm{l}^{-1}$ humic acid

Table (4) Effect of humic , ascorbic acid, cultivar and their interactions on leaves number of olive transplant cvs. 'Khithairy ' and ' Sorany'.

\begin{tabular}{|c|c|c|c|c|c|c|c|}
\hline \multirow[b]{2}{*}{ 竎 } & \multirow{2}{*}{ 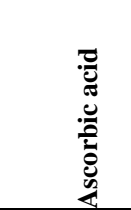 } & \multicolumn{4}{|c|}{ Humic concentration } & \multirow{2}{*}{$\begin{array}{l}\text { cultivar } \\
\text { ×ascorbic } \\
\text { acid }\end{array}$} & \multirow{2}{*}{$\begin{array}{l}\text { cultivar } \\
\text { effect }\end{array}$} \\
\hline & & $\mathbf{0}$ & 20 & 40 & 60 & & \\
\hline \multirow{4}{*}{ 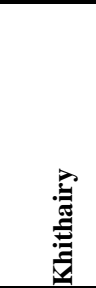 } & $\mathbf{0}$ & $\begin{array}{l}87.67 \\
0\end{array}$ & $\begin{array}{l}185.33 \\
b\end{array}$ & $\begin{array}{l}190.33 \\
\mathrm{a}\end{array}$ & $\begin{array}{l}191.33 \\
\mathrm{a}\end{array}$ & $\begin{array}{l}163.67 \\
\mathrm{c}\end{array}$ & \multirow{4}{*}{$\begin{array}{l}166.71 \\
\mathrm{a}\end{array}$} \\
\hline & 500 & $\begin{array}{l}166.33 \\
\text { de }\end{array}$ & $\begin{array}{l}175.00 \\
\mathrm{c}\end{array}$ & $\begin{array}{l}175.67 \\
\mathrm{c}\end{array}$ & $\begin{array}{l}175.00 \\
\mathrm{c}\end{array}$ & $\begin{array}{l}173.00 \\
\mathrm{a}\end{array}$ & \\
\hline & 1000 & $\begin{array}{l}163.67 \\
\mathrm{e}\end{array}$ & $\begin{array}{l}175.00 \\
\mathrm{c}\end{array}$ & $\begin{array}{l}172.00 \\
\mathrm{c}\end{array}$ & $\begin{array}{l}170.67 \\
\mathrm{~cd}\end{array}$ & $\begin{array}{l}170.33 \\
\mathrm{~b}\end{array}$ & \\
\hline & 2000 & $\begin{array}{l}143.00 \\
\mathrm{f}\end{array}$ & $\begin{array}{l}164.67 \\
\mathrm{e}\end{array}$ & $\begin{array}{l}166.67 \\
\text { de }\end{array}$ & $\begin{array}{l}165.00 \\
\mathrm{e}\end{array}$ & $\begin{array}{l}159.83 \\
\mathrm{~d}\end{array}$ & \\
\hline \multirow[b]{4}{*}{ 胥 } & 0 & $\begin{array}{l}65.33 \\
q\end{array}$ & $\begin{array}{l}80.67 \\
\mathrm{p}\end{array}$ & $\begin{array}{l}95.00 \\
\mathrm{n}\end{array}$ & $\begin{array}{l}105.33 \\
\mathrm{~m}\end{array}$ & $\begin{array}{l}86.58 \\
\mathrm{~h} \\
\end{array}$ & \multirow{4}{*}{$\begin{array}{l}113.58 \\
\mathrm{~b}\end{array}$} \\
\hline & 500 & $\begin{array}{l}133.67 \\
\mathrm{gh}\end{array}$ & $\begin{array}{l}105.00 \\
\mathrm{~m}\end{array}$ & $\begin{array}{l}113.00 \\
\mathrm{kl}\end{array}$ & $\begin{array}{l}122.33 \\
\mathrm{ij}\end{array}$ & $\begin{array}{l}118.50 \\
\mathrm{~g}\end{array}$ & \\
\hline & 1000 & $\begin{array}{l}137.33 \\
\mathrm{~g}\end{array}$ & $\begin{array}{l}109.00 \\
\operatorname{lm}\end{array}$ & $\begin{array}{l}117.33 \\
\mathrm{jk}\end{array}$ & $\begin{array}{l}124.33 \\
\mathrm{i}\end{array}$ & $\begin{array}{l}122.00 \\
\mathrm{f}\end{array}$ & \\
\hline & 2000 & $\begin{array}{l}145.00 \\
\mathrm{f}\end{array}$ & $\begin{array}{l}113.67 \\
\mathrm{kl}\end{array}$ & $\begin{array}{l}120.33 \\
\mathrm{ij}\end{array}$ & $\begin{array}{l}130.00 \\
\mathrm{~h}\end{array}$ & $\mathrm{e}^{127.25}$ & \\
\hline \multicolumn{2}{|c|}{ Humic effect } & $\begin{array}{l}130.25 \\
\mathrm{~d}\end{array}$ & $\begin{array}{l}138.54 \\
\mathrm{c}\end{array}$ & $\begin{array}{l}143.79 \\
\mathrm{~b}\end{array}$ & $\begin{array}{l}148.00 \\
\mathrm{a}\end{array}$ & \multirow{3}{*}{$\begin{array}{l}\text { Ascorbic } \\
\text { acid effect }\end{array}$} & \\
\hline \multirow{2}{*}{ كُّ } & Khithairy & $\begin{array}{l}140.17 \\
\mathrm{~b}\end{array}$ & $\begin{array}{l}175.00 \\
\mathrm{a}\end{array}$ & $\begin{array}{l}176.17 \\
\mathrm{a}\end{array}$ & $\begin{array}{l}175.50 \\
\mathrm{a}\end{array}$ & & \\
\hline & Sorany & $\begin{array}{l}120.33 \\
\mathrm{c}\end{array}$ & $\begin{array}{l}102.08 \\
\mathrm{e}\end{array}$ & $\begin{array}{l}111.42 \\
\mathrm{~d}\end{array}$ & $\begin{array}{l}120.50 \\
\mathrm{c}\end{array}$ & & \\
\hline \multirow{4}{*}{ 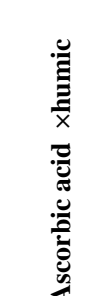 } & 0 & $\begin{array}{l}76.5 \\
h\end{array}$ & $\begin{array}{l}133.000 \\
\mathrm{~g}\end{array}$ & $\begin{array}{l}142.667 \\
\text { b-d }\end{array}$ & $\begin{array}{l}148.333 \\
\mathrm{ab}\end{array}$ & $\begin{array}{l}125.13 \\
\mathrm{c}\end{array}$ & \\
\hline & 500 & $\begin{array}{l}150.00 \\
\mathrm{a}\end{array}$ & $\begin{array}{l}140.000 \\
\text { ef }\end{array}$ & $\begin{array}{l}144.333 \\
\text { cd }\end{array}$ & $\begin{array}{l}148.667 \\
\mathrm{a}\end{array}$ & $\begin{array}{l}145.75 \\
\mathrm{a}\end{array}$ & \\
\hline & 1000 & $\begin{array}{l}150.50 \\
\mathrm{a}\end{array}$ & $\begin{array}{l}142.000 \\
\text { d-f }\end{array}$ & $\begin{array}{l}144.667 \\
\text { b-d }\end{array}$ & $\begin{array}{l}147.500 \\
\text { a-c }\end{array}$ & $\begin{array}{l}146.17 \\
\mathrm{a}\end{array}$ & \\
\hline & 2000 & $\begin{array}{l}144.00 \\
\mathrm{~cd}\end{array}$ & $\begin{array}{l}139.167 \\
\mathrm{f}\end{array}$ & $\begin{array}{l}143.500 \\
\text { de }\end{array}$ & $\begin{array}{l}147.500 \\
a-c\end{array}$ & $\begin{array}{l}143.54 \\
b\end{array}$ & \\
\hline
\end{tabular}

Means within a column, row and their interactions followed with the same letters are not significantly different from each others according to Duncans multiple ranges test at $5 \%$ level.

Application of ascorbic acid at 1000mg. $1^{-1}$ significantly increased leaf number (146.17) when compared with other concentration. Results of cultivars revealed that Khithairy gave the highest value of leaf number (166.71) when compared with Sorany cultivar (113.58). The interaction between humic acid and ascorbic acid notes that the highest leaves number per transplant (150.50) was observed in transplants received 0mg. $1^{-1}$ humic acid and $1000 \mathrm{mg} . \mathrm{l}^{-1}$ ascorbic acid and the lowest leaves number (76.50) recorded in untreated transplants. 
Khithairy cultivar when treated with $500 \mathrm{mg} \cdot \mathrm{l}^{-1}$ ascorbic acid had significantly the highest number of leaves (173.00) per transplant when compared to the other interaction and the lowest number of leaves (86.58) recorded in untreated Sorany cultivar. Whereas the interaction between humic acid and Cultivar showed that the Khithairy cultivar when treated with $40 \mathrm{mg} . \mathrm{l}^{-1}$ humic acid gave the highest value leaves number (176.17) per transplant and the lowest value (102.08) was recorded in Sorany cultivar when treated with $20 \mathrm{mg} \cdot \mathrm{l}^{-1}$ humic acid.

Leaves number per transplant was significantly increasing by humic acid, ascorbic acid and Cultivar interaction, Sorany transplant foliar sprayed with $60 \mathrm{mg} .1^{-1}$ humic acid and $0 \mathrm{mg} . .^{-1}$ ascorbic acid produced the highest number of leaves (191.33) whereas the untreated Sorany transplant showed the lowest leaves number per transplant (65.33).

\subsection{Shoot length:}

Table (5) showed that the shoot length per plant increased significantly with increased humic acid level application, when transplants treated with $60 \mathrm{mg} .1^{-1}$ humic acid gave the highest value (48.50).

For the application of ascorbic acid show that when the transplant treated by $2000 \mathrm{mg} \cdot \mathrm{l}^{-1}$ ascorbic acid gave the highest value (44.83) when compared with other concentration. Whereas the lowest value (39.88) was recorded when transplant treated with 500mg. $\mathrm{l}^{-1}$ ascorbic acid.

Table (5) Effect of humic ,ascorbic acid, cultivar and their interactions on lateral shoot length average of olive transplant cvs. 'Khithairy ' and ' Sorany'.

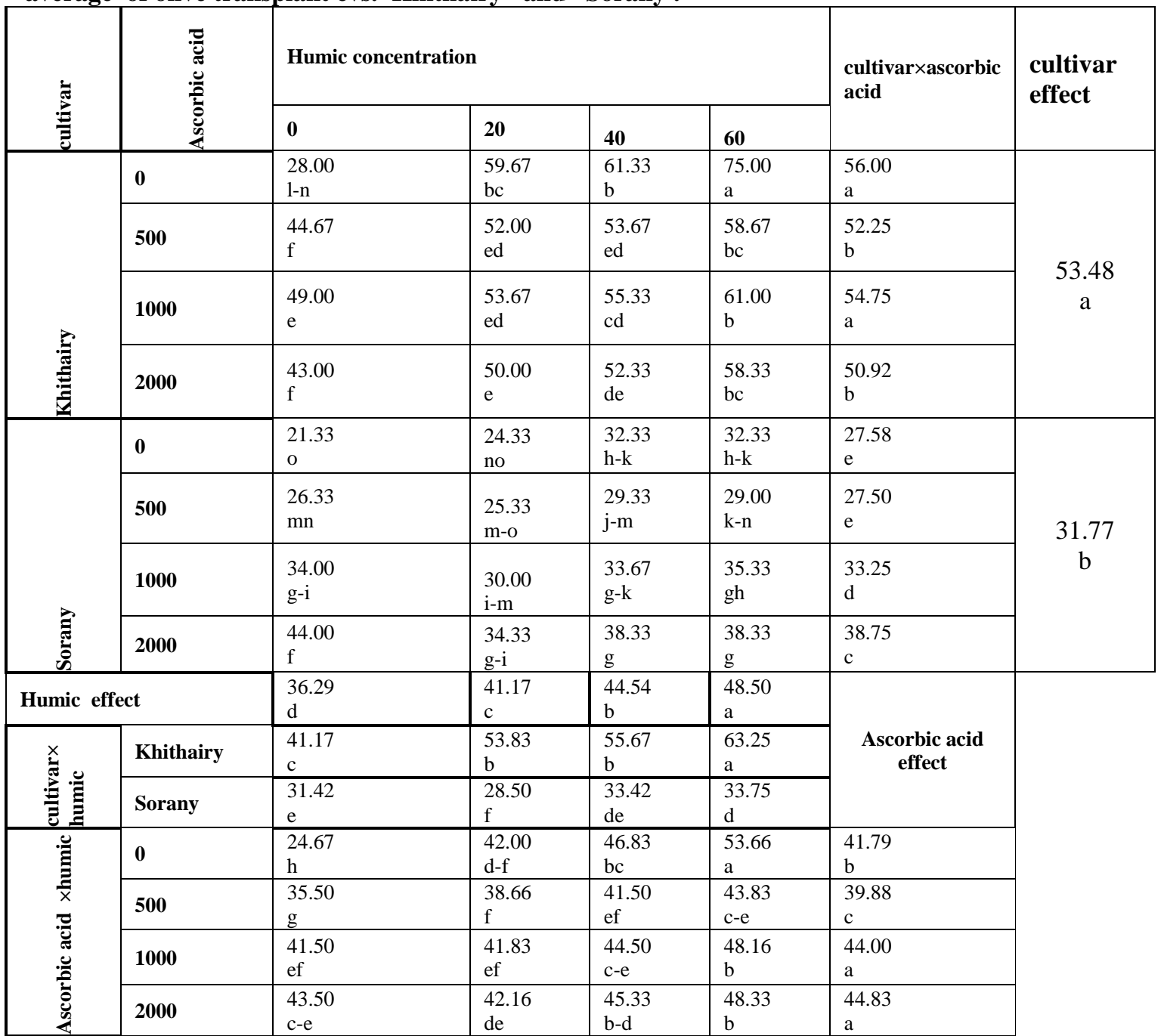

Means within a column, row and their interactions followed with the same letters are not significantly different from each other's according to Duncan's multiple ranges test at $5 \%$ level.

The shoot length was significantly influenced by cultivars, Khithairy transplants had around (53.48) that highest than Sorany cultivar. The interaction between humic acid and ascorbic acid show that the transplant when treated only by $60 \mathrm{mg} .1^{-1}$ humic acid gave the highest value $(53.66 \mathrm{~cm})$ of shoot length compared with other interactions and the lowest value (24.67) was recorded in untreated transplant.The interaction between ascorbic acid and cultivar showed that untreated Khithairy transplant gave the highest value when compared with other 
interaction, and the lowest shoot length (27.50) recorded in Sorany cultivar when treated with500mg. $1^{-1}$ ascorbic acid.

Also for the interaction between humic acid and Cultivar showed that the Khithairy transplant gave the highest value $(63.25 \mathrm{~cm})$ when compared with other interaction. The results for the interaction between humic acid, ascorbic acid and Cultivar significantly influenced shoot length, the highest shoot length $(75.00 \mathrm{~cm})$ was observed in Khithairy transplant that treated by $60 \mathrm{mg} \cdot \mathrm{l}^{-1}$ humic acid $+0 \mathrm{mg} \cdot \mathrm{l}^{-1}$ ascorbic acid.

\subsection{Leaf area cm2:}

Table (6) showed that the olive transplants were spraying with humic acid $60 \mathrm{mg} \cdot \mathrm{l}^{-1}$ concentration gave the highest leaf area $\left(4.84 \mathrm{~cm}^{2}\right)$ when compared with the control.

Table (6) Effect of humic ascorbic acid, cultivar and their interactions on leaf area of olive transplant cvs. 'Khithairy ' and ' Sorany'.

\begin{tabular}{|c|c|c|c|c|c|c|c|}
\hline \multirow[b]{2}{*}{ 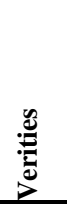 } & \multirow{2}{*}{ 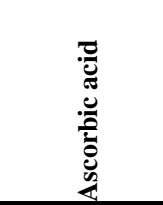 } & \multicolumn{4}{|c|}{ Humic concentration } & \multirow{2}{*}{$\begin{array}{l}\text { cultivar } \\
\text { ×ascorbic } \\
\text { acid }\end{array}$} & \multirow{2}{*}{$\begin{array}{l}\text { cultivar } \\
\text { effect }\end{array}$} \\
\hline & & $\mathbf{0}$ & 20 & 40 & 60 & & \\
\hline \multirow{4}{*}{ 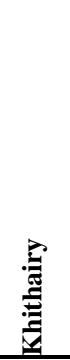 } & $\mathbf{0}$ & $\begin{array}{l}4.58 \\
\mathrm{f}\end{array}$ & $\begin{array}{l}5.88 \\
\mathrm{~d}\end{array}$ & $\begin{array}{l}6.14 \\
d\end{array}$ & $\begin{array}{l}7.60 \\
a b\end{array}$ & $\begin{array}{l}6.05 \\
b\end{array}$ & \multirow{4}{*}{$\begin{array}{l}5.79 \\
\mathrm{a}\end{array}$} \\
\hline & 500 & $\begin{array}{l}6.35 \\
\mathrm{~cd}\end{array}$ & $\begin{array}{l}6.38 \\
\mathrm{~cd}\end{array}$ & $\begin{array}{l}7.01 \\
\mathrm{bc}\end{array}$ & $\begin{array}{l}7.67 \\
a\end{array}$ & $\begin{array}{l}6.85 \\
\mathrm{a}\end{array}$ & \\
\hline & 1000 & $\begin{array}{l}3.73 \\
\text { gh }\end{array}$ & $\begin{array}{l}5.03 \\
\mathrm{f}\end{array}$ & $\begin{array}{l}5.97 \\
d\end{array}$ & $\begin{array}{l}6.02 \\
d\end{array}$ & $\begin{array}{l}5.19 \\
\mathrm{c}\end{array}$ & \\
\hline & 2000 & $\begin{array}{l}3.50 \\
g-i\end{array}$ & $\begin{array}{l}5.17 \\
\text { ef }\end{array}$ & $\begin{array}{l}5.73 \\
\text { de }\end{array}$ & $\begin{array}{l}5.81 \\
\text { de }\end{array}$ & $\begin{array}{l}5.05 \\
\mathrm{c}\end{array}$ & \\
\hline \multirow{4}{*}{ त्் } & $\mathbf{0}$ & $\begin{array}{l}2.04 \\
\mathrm{n}\end{array}$ & $\begin{array}{l}2.68 \\
\text { k-n }\end{array}$ & $\begin{array}{l}3.22 \\
\mathrm{~g}-\mathrm{k}\end{array}$ & $\begin{array}{l}2.85 \\
\mathrm{i}-\mathrm{m}\end{array}$ & $\begin{array}{l}2.70 \\
\mathrm{e}\end{array}$ & \multirow{4}{*}{$\begin{array}{l}2.95 \\
b\end{array}$} \\
\hline & 500 & $\begin{array}{l}2.40 \\
1-n\end{array}$ & $\begin{array}{l}2.55 \\
\text { k-n }\end{array}$ & $\begin{array}{l}2.29 \\
\mathrm{mn}\end{array}$ & $\begin{array}{l}2.37 \\
1-n\end{array}$ & $\begin{array}{l}2.40 \\
\mathrm{f}\end{array}$ & \\
\hline & 1000 & $\begin{array}{l}2.65 \\
k-n\end{array}$ & $\begin{array}{l}2.59 \\
\text { k-n }\end{array}$ & $\begin{array}{l}3.10 \\
\mathrm{~h}-1\end{array}$ & $\begin{array}{l}2.79 \\
j-m\end{array}$ & $\begin{array}{l}2.78 \\
\mathrm{e}\end{array}$ & \\
\hline & 2000 & $\begin{array}{l}4.76 \\
\mathrm{f} \\
\end{array}$ & $\begin{array}{l}3.46 \\
g-j\end{array}$ & $\begin{array}{l}3.84 \\
\mathrm{~g} \\
\end{array}$ & $\begin{array}{l}3.62 \\
\mathrm{gh}\end{array}$ & $\begin{array}{l}3.92 \\
\mathrm{~d} \\
\end{array}$ & \\
\hline & Humic effect & $\begin{array}{l}3.75 \\
\mathrm{c}\end{array}$ & $\begin{array}{l}4.22 \\
b\end{array}$ & $\begin{array}{l}4.66 \\
a\end{array}$ & $\begin{array}{l}4.84 \\
\mathrm{a}\end{array}$ & \multirow{3}{*}{$\begin{array}{l}\text { Ascorbic acid } \\
\text { effect }\end{array}$} & \\
\hline \multirow{2}{*}{ 﨎 } & Khithairy & $\begin{array}{l}4.54 \\
\mathrm{~d}\end{array}$ & $\begin{array}{l}5.62 \\
\mathrm{c}\end{array}$ & $\begin{array}{l}6.21 \\
b\end{array}$ & $\begin{array}{l}6.77 \\
a\end{array}$ & & \\
\hline & Sorany & $\begin{array}{l}2.96 \\
\mathrm{e}\end{array}$ & $\begin{array}{l}2.82 \\
\mathrm{e}\end{array}$ & $\begin{array}{l}3.11 \\
\mathrm{e}^{2}\end{array}$ & $\begin{array}{l}2.91 \\
\mathrm{e}\end{array}$ & & \\
\hline \multirow{4}{*}{ 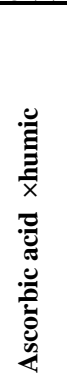 } & $\mathbf{0}$ & $\begin{array}{l}3.31 \\
\mathrm{f}\end{array}$ & $\begin{array}{l}4.28 \\
\mathrm{~cd}\end{array}$ & $\begin{array}{l}4.68 \\
b c\end{array}$ & $\begin{array}{l}5.22 \\
\mathrm{a}\end{array}$ & $\begin{array}{l}4.37 \\
\mathrm{~b}\end{array}$ & \\
\hline & 500 & $\begin{array}{l}4.38 \\
\mathrm{~cd}\end{array}$ & $\begin{array}{l}4.46 \\
\mathrm{~cd}\end{array}$ & $\begin{array}{l}4.648 \\
\text { b c }\end{array}$ & $\begin{array}{l}5.02 \\
\mathrm{ab}\end{array}$ & $\begin{array}{l}4.63 \\
\mathrm{a}\end{array}$ & \\
\hline & 1000 & $\begin{array}{l}3.19 \\
\mathrm{f}\end{array}$ & $\begin{array}{l}3.81 \\
\mathrm{e}\end{array}$ & $\begin{array}{l}4.53 \\
b-d\end{array}$ & $\begin{array}{l}4.40 \\
\mathrm{~cd}\end{array}$ & $\begin{array}{l}3.99 \\
\mathrm{c}\end{array}$ & \\
\hline & 2000 & $\begin{array}{l}4.13 \\
\mathrm{de}\end{array}$ & $\begin{array}{l}4.31 \\
\mathrm{~cd}\end{array}$ & $\begin{array}{l}4.78 \\
a-c\end{array}$ & $\begin{array}{l}4.71 \\
\mathrm{bc}\end{array}$ & $\begin{array}{l}4.49 b \\
a\end{array}$ & \\
\hline
\end{tabular}

Means within a column, row and their interactions followed with the same letters are not significantly different from each other's according to Duncan's multiple ranges test at $5 \%$ level.

When the olive transplants treated with ascorbic acid concentration in $500 \mathrm{mg} \cdot \mathrm{l}^{-1}$ gave the highest value $\left(4.63 \mathrm{~cm}^{2}\right)$ when compared with the other concentrations. Results of cultivars revealed that Khithairy cultivar had significant effect on the leaf area $\left(5.79 \mathrm{~cm}^{2}\right)$ when compared with Sorany olive cultivar $\left(2.95 \mathrm{~cm}^{2}\right)$.

Results indicated that the combination between ascorbic acid and humic acid concentration displayed that $0 \mathrm{mg} . \mathrm{l}^{-1}$ ascorbic acid and $60 \mathrm{mg} . \mathrm{l}^{-1}$ humic acid are appeared to be the most potent treatments as it gave the 
highest leaf area value $\left(5.22 \mathrm{~cm}^{2}\right)$. The interaction between ascorbic acid and cultivar significantly influenced of leaf area, we notice that the Khithairy transplant when treated with $500 \mathrm{mg} .1^{-1}$ ascorbic acid gave the highest value of leaf area $\left(6.85 \mathrm{~cm}^{2}\right)$ and the lowest value $\left(2.40 \mathrm{~cm}^{2}\right)$ recorded in Sorany transplants when treated by $500 \mathrm{mg} .1^{-1}$ ascorbic acid . The interaction between cultivar and humic acid caused significant difference in leaf area, the highest value of leaf area $\left(6.77 \mathrm{~cm}^{2}\right)$ was recorded in Khithairy transplant when treated with $60 \mathrm{mg} .1^{-1}$ humic acid , and the lowest value $\left(2.82 \mathrm{~cm}^{2}\right)$ was recorded in Sorany transplants when treated by $20 \mathrm{mg} \cdot .^{-1}$ humic acid . The interaction between humic, ascorbic acid and cultivar caused significant difference in leaf area, the highest value $\left(7.67 \mathrm{~cm}^{2}\right)$ was recorded in Khithairy transplants when treated with $60 \mathrm{mg} \cdot \mathrm{l}^{-1}$ of humic acid and $500 \mathrm{mg} \cdot \mathrm{l}^{-1}$ ascorbic acid and the lowest value $\left(2.04 \mathrm{~cm}^{2}\right)$ was recorded in untreated Sorany cultivar.

\subsection{Lateral shoot number;}

The obtained results of table (7) revealed that spraying olive transplants with humic concentrations resulted in a significant increase in lateral shoot number particularly at $60 \mathrm{mg} \cdot \mathrm{l}^{-1}$ level as compared to the control

Table (7) Effect of humic, ascorbic acid, cultivar and their interactions on lateral shoot number of olive transplant cvs. 'Khithairy ' and ' Sorany'.

\begin{tabular}{|c|c|c|c|c|c|c|c|}
\hline \multirow[b]{2}{*}{ : } & \multirow{2}{*}{ 递 } & \multicolumn{4}{|c|}{ Humic concentration } & \multirow{2}{*}{$\begin{array}{l}\text { Cultivar } \\
\text { ×ascorbic } \\
\text { acid } \\
\end{array}$} & \multirow[b]{2}{*}{$\begin{array}{l}\text { cultivar } \\
\text { effect }\end{array}$} \\
\hline & & $\mathbf{0}$ & 20 & 40 & 60 & & \\
\hline \multirow{4}{*}{ 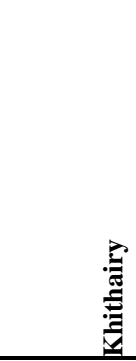 } & $\mathbf{0}$ & $\begin{array}{l}3 \\
\mathrm{i}-\mathrm{k} \\
\end{array}$ & $\begin{array}{l}3.33 \\
h-j \\
\end{array}$ & $\begin{array}{l}5 \\
\mathrm{c}-\mathrm{e} \\
\end{array}$ & $\begin{array}{l}8 \\
\mathrm{a} \\
\end{array}$ & $\begin{array}{l}4.83 \\
\mathrm{~b} \\
\end{array}$ & \multirow[t]{4}{*}{$\begin{array}{c}4.98 \\
\mathrm{a}\end{array}$} \\
\hline & 500 & $\begin{array}{l}7.33 \\
\mathrm{a} \\
\end{array}$ & $\begin{array}{l}5.67 \\
\mathrm{bc}\end{array}$ & $\begin{array}{l}6 \\
\mathrm{~b} \\
\end{array}$ & $\begin{array}{l}8 \\
\mathrm{a} \\
\end{array}$ & $\begin{array}{l}6.75 \\
\mathrm{a} \\
\end{array}$ & \\
\hline & 1000 & $\begin{array}{l}4.33 \\
\mathrm{e}-\mathrm{g} \\
\end{array}$ & $\begin{array}{l}4 \\
\mathrm{f}-\mathrm{h} \\
\end{array}$ & $\begin{array}{l}5 \\
\mathrm{c}-\mathrm{e} \\
\end{array}$ & $\begin{array}{l}6 \\
\mathrm{~b} \\
\end{array}$ & $\begin{array}{l}4.83 \\
\mathrm{~b} \\
\end{array}$ & \\
\hline & 2000 & $\begin{array}{l}2.33 \\
\mathrm{k} \\
\end{array}$ & $\begin{array}{l}2.67 \\
\mathrm{jk} \\
\end{array}$ & $\begin{array}{l}3.67 \\
\mathrm{~g}-\mathrm{i} \\
\end{array}$ & $\begin{array}{l}5.33 \\
\text { b-d } \\
\end{array}$ & $\begin{array}{l}3.5 \\
\mathrm{e} \\
\end{array}$ & \\
\hline \multirow[b]{4}{*}{ 苞 } & 0 & $\begin{array}{l}3.67 \\
\text { g-i }\end{array}$ & $\begin{array}{l}4.33 \\
\mathrm{e}-\mathrm{g}\end{array}$ & $\begin{array}{l}5.33 \\
\text { b-d }\end{array}$ & $\begin{array}{l}4.33 \\
\mathrm{e}-\mathrm{g}\end{array}$ & $\begin{array}{l}4.42 \\
\mathrm{c}\end{array}$ & \multirow[t]{4}{*}{$\begin{array}{c}4.31 \\
\mathrm{~b}\end{array}$} \\
\hline & 500 & $\begin{array}{l}3.33 \\
\mathrm{~h}-\mathrm{j} \\
\end{array}$ & $\begin{array}{l}4 \\
\mathrm{f}-\mathrm{h} \\
\end{array}$ & $\begin{array}{l}4 \\
\mathrm{f}-\mathrm{h} \\
\end{array}$ & $\begin{array}{l}4 \\
\mathrm{f}-\mathrm{h} \\
\end{array}$ & $\begin{array}{l}3.83 \\
\mathrm{de} \\
\end{array}$ & \\
\hline & 1000 & $\begin{array}{l}3.33 \\
\mathrm{~h}-\mathrm{j} \\
\end{array}$ & $\begin{array}{l}4 \\
\mathrm{f}-\mathrm{h} \\
\end{array}$ & $\begin{array}{l}4 \\
\mathrm{f}-\mathrm{h} \\
\end{array}$ & $\begin{array}{l}4.33 \\
\mathrm{e}-\mathrm{g} \\
\end{array}$ & $\begin{array}{l}3.92 \\
\mathrm{~d} \\
\end{array}$ & \\
\hline & 2000 & $\begin{array}{l}5.33 \\
\text { b-d } \\
\end{array}$ & $\begin{array}{l}5 \\
\mathrm{c}-\mathrm{e} \\
\end{array}$ & $\begin{array}{l}5.33 \\
\text { b-d } \\
\end{array}$ & $\begin{array}{l}4.67 \\
\text { d-f }\end{array}$ & $\begin{array}{l}5.08 \\
\mathrm{~b}\end{array}$ & \\
\hline \multicolumn{2}{|c|}{ Humic effect } & $\begin{array}{l}4.08 \\
\mathrm{c} \\
\end{array}$ & $\begin{array}{l}4.13 \\
\mathrm{c} \\
\end{array}$ & $\begin{array}{l}4.79 \\
\mathrm{~b} \\
\end{array}$ & $\begin{array}{l}5.58 \\
\mathrm{a} \\
\end{array}$ & \multirow[b]{3}{*}{$\begin{array}{l}\text { ascorbic acid } \\
\text { effect }\end{array}$} & \\
\hline \multirow{2}{*}{ 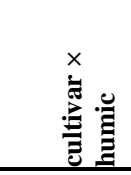 } & Khithairy & $\begin{array}{l}4.25 \\
\mathrm{~d} \\
\end{array}$ & $\begin{array}{l}3.92 \\
\mathrm{~d} \\
\end{array}$ & $\begin{array}{l}4.92 \\
\mathrm{~b} \\
\end{array}$ & $\begin{array}{l}6.83 \\
\mathrm{a} \\
\end{array}$ & & \\
\hline & Sorany & $\begin{array}{l}3.92 \\
\mathrm{~d} \\
\end{array}$ & $\begin{array}{l}4.33 \\
\mathrm{~cd}\end{array}$ & $\begin{array}{l}4.67 \\
\mathrm{bc} \\
\end{array}$ & $\begin{array}{l}4.33 \\
\mathrm{~cd}\end{array}$ & & \\
\hline \multirow{4}{*}{ 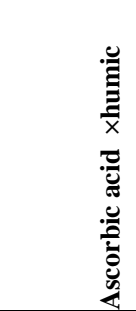 } & $\mathbf{0}$ & $\begin{array}{l}3.333 \\
\mathrm{f} \\
\end{array}$ & $\begin{array}{l}3.83 \\
\text { ef }\end{array}$ & $\begin{array}{l}5.16 \\
\mathrm{~b} \\
\end{array}$ & $\begin{array}{l}6.16 \\
\mathrm{a} \\
\end{array}$ & $\begin{array}{l}4.63 \\
\mathrm{~b} \\
\end{array}$ & \\
\hline & 500 & $5.33 b$ & $4.83 \mathrm{bc}$ & $5 \mathrm{bc}$ & $6 a$ & $5.29 \mathrm{a}$ & \\
\hline & 1000 & $3.83 \mathrm{ef}$ & 4 de & $4.5 \mathrm{~cd}$ & $5.16 \mathrm{~b}$ & $4.38 \mathrm{bc}$ & \\
\hline & 2000 & $3.83 \mathrm{ef}$ & 3.83 ef & $4.5 \mathrm{~cd}$ & $5 \mathrm{bc}$ & $4.29 \mathrm{c}$ & \\
\hline
\end{tabular}

Means within a column, row and their interactions followed with the same letters are not

significantly different from each other's according to Duncan's multiple ranges test at $5 \%$ level.

. Olive transplants treated with 500mg. $1^{-1}$ ascorbic gave the highest value (5.29) and the lowest value (4.29) was recorded in the olive transplants when treated with $2000 \mathrm{mg} . \mathrm{l}^{-1}$ ascorbic acid. Results of cultivars revealed that Khithairy gave the highest lateral shoot number (4.98) compared with the Sorany olive cultivar (4.31). 
Results indicated that the combination between humic and ascorbic acid concentration displayed that $0 \mathrm{mg} \cdot \mathrm{l}^{-1}$ Ascorbic acid and $60 \mathrm{mg} . \mathrm{l}^{-1}$ humic acid gave the highest value of lateral shoot number (6.167) and the lowest value (3.33) was recorded in untreated olive transplants. Results of cultivar and humic concentration interaction revealed that spraying Khithairy with humic at the rate of $60 \mathrm{mg} \cdot \mathrm{l}^{-1}$ gave the highest value (6.83) and the lowest value (3.92) recorded in untreated Sorany cultivar, and Khithairy cultivar when treated with 20 mg. $1^{-1}$ humic acid. Results of cultivar and ascorbic acid concentration interaction revealed that spraying Khithairy with ascorbic acid at the rate of $500 \mathrm{mg}^{-1}{ }^{-1}$ gave the highest lateral shoot number (6.75) and the lowest number of lateral shoot number (3.50) recorded in Khithairy transplants when treated with $2000 \mathrm{mg} \cdot \mathrm{l}^{-1}$ ascorbic acid. Results ascorbic acid and humic acid and cultivar interaction revealed that spraying Khithairy olive cultivar with $500 \mathrm{mg} \cdot \mathrm{l}^{-1}$ ascorbic acid plus $60 \mathrm{mg} \cdot \mathrm{l}^{-1}$ humic acid gave the highest value of lateral shoot number (8.0) also the Khithairy olive with $0 \mathrm{mg} . \mathrm{l}^{-1}$ ascorbic plus $60 \mathrm{mg} \cdot \mathrm{l}^{-1}$ humic gave (8.0) lateral shoot number.

\subsection{Chlorophyll\%}

In table (8) showed foliar application of humic acid increased significantly chlorophyll content, the highest value of chlorophyll content (72.51\%) recorded in the olive transplants when spray with $40 \mathrm{mg} . \mathrm{l}^{-1}$, and the lowest value $\left(68.71 \%\right.$ ) was recorded in untreated olive transplants Application of ascorbic acid at $1000 \mathrm{mg} . \mathrm{l}^{-1}$ increased significantly chlorophyll percentage (72.15) when compared with the control that that gave the lowest value $(68.17 \%)$.

Table (8) Effect of humic, ascorbic acid, cultivar and their interactions on chlorophyll \% of olive transplant cvs. 'Khithairy ' and 'Sorany'.

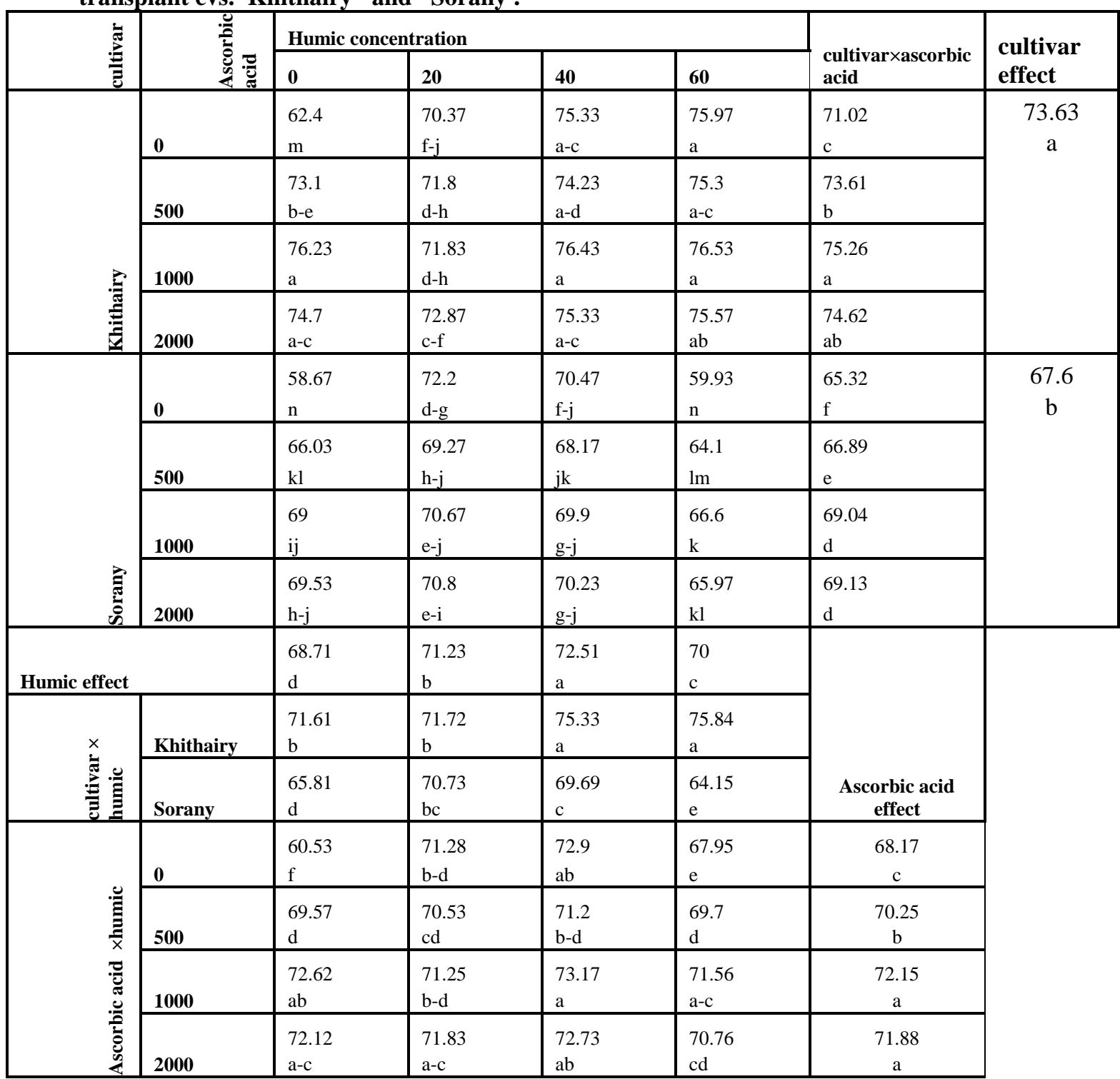

Means within a column, row and their interactions followed with the same letters are not significantly different from each other's according to Duncan's multiple ranges test at $5 \%$ level. 
Total chlorophyll percentage in leaves differed significantly between the two cultivars. Khithairy cultivar contained significantly higher chlorophyll percentage (73.63\%) compared with leaves of Sorany cultivar $(67.60 \%)$.

Regarding the effect of ascorbic acid and humic interaction chlorophyll percentage increased significantly in olive transplants treated with $40 \mathrm{mg} . \mathrm{l}^{-1}$ humic acid and $1000 \mathrm{mg} . \mathrm{l}^{-1}$ ascorbic acid (73.167\%) as compared with the control that gave the lowest value $(60.53 \%)$.

Results of humic and cultivars interaction revealed that spraying Khithairy olive cultivar with $60 \mathrm{mg} . \mathrm{l}^{-1}$ gave the highest value of chlorophyll percentage $(75.84 \%)$ and the lowest value was recorded in Sorany olive cultivar with $60 \mathrm{mg} .1^{-1}$ humic $(64.15 \%)$. Khithairy cultivar when treated with $1000 \mathrm{mg} . \mathrm{l}^{-1}$ ascorbic acid gave the highest value (75.26\%), the lowest value of chlorophyll \% was recorded in untreated Sorany cultivar $(65.32 \%)$ . Results of ascorbic acid, humic acid and cultivars interaction indicated that spraying Khithairy olive cultivar with $1000 \mathrm{mg} . \mathrm{l}^{-1}$ ascorbic acid and $60 \mathrm{mg} . \mathrm{l}^{-1}$ humic acid was the most potent treatment which gave $(76.53 \%)$, while the lowest value of chlorophyll percentage coincided with untreated Sorany cultivar (58.67\%).

\subsection{Leaves fresh weight;}

In table (9) notice that the foliar application of humic acid increased leaves fresh weight of olive transplants, the highest leaves fresh weight was produced by transplants treated with $20 \mathrm{mg} . \mathrm{l}^{-1}$ humic acid (14.75)g when compared with other concentrations. Application of ascorbic acid increased leaves fresh weight , the highest value was produced by olive transplants treated with $2000 \mathrm{mg} .1^{-1}$ ascorbic acid that gave (15.44) gm, and the lowest value (11.30)g was recorded in untreated olive transplants

Table (9) Effect of humic ascorbic acid, cultivar and their interactions on leaves fresh weight olive transplant cvs. 'Khithairy ' and ' Sorany'.

\begin{tabular}{|c|c|c|c|c|c|c|c|}
\hline \multirow{2}{*}{ 昙 } & \multirow{2}{*}{ 象 } & \multicolumn{4}{|c|}{ Humic concentration } & \multirow{2}{*}{$\begin{array}{l}\text { cultivar } \times \\
\text { ascorbic acid }\end{array}$} & \multirow{2}{*}{$\begin{array}{l}\text { cultivar } \\
\text { effect }\end{array}$} \\
\hline & & $\mathbf{0}$ & 20 & 40 & 60 & & \\
\hline \multirow{4}{*}{ 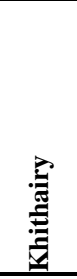 } & 0 & $\begin{array}{c}10.67 \\
\mathrm{kl} \\
\end{array}$ & $\begin{array}{c}20.20 \\
\text { a-c } \\
\end{array}$ & $\begin{array}{c}18.13 \\
\text { ef }\end{array}$ & $\begin{array}{c}13.57 \\
\mathrm{i} \\
\end{array}$ & $\begin{array}{c}15.64 \\
\mathrm{~d}\end{array}$ & \multirow{4}{*}{$\begin{array}{c}17.82 \\
\mathrm{a}\end{array}$} \\
\hline & 500 & $\begin{array}{c}16.27 \\
\mathrm{gh}\end{array}$ & $\begin{array}{c}18.73 \\
\text { de }\end{array}$ & $\begin{array}{c}17.63 \\
\text { ef }\end{array}$ & $\begin{array}{c}15.30 \\
\mathrm{~h}\end{array}$ & $\begin{array}{c}16.98 \\
\mathrm{c}\end{array}$ & \\
\hline & 1000 & $\begin{array}{c}19.43 \\
\text { cd }\end{array}$ & $\begin{array}{c}19.90 \\
\text { bc }\end{array}$ & $\begin{array}{c}18.43 \\
\text { de }\end{array}$ & $\begin{array}{c}17.03 \\
\text { fg }\end{array}$ & $\begin{array}{c}18.70 \\
b\end{array}$ & \\
\hline & 2000 & $\begin{array}{c}21.17 \\
\mathrm{a} \\
\end{array}$ & $\begin{array}{c}20.73 \\
\mathrm{ab}\end{array}$ & $\begin{array}{c}19.87 \\
\text { bc }\end{array}$ & $\begin{array}{c}18.00 \\
\text { ef }\end{array}$ & $\begin{array}{c}19.94 \\
\mathrm{a} \\
\end{array}$ & \\
\hline \multirow[b]{4}{*}{ 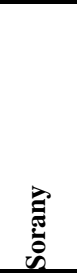 } & 0 & $\begin{array}{c}2.87 \\
\mathrm{q} \\
\end{array}$ & $\begin{array}{c}10.23 \\
1 \\
\end{array}$ & $\begin{array}{c}9.13 \\
\mathrm{~m} \\
\end{array}$ & $\begin{array}{c}5.63 \\
\mathrm{p}\end{array}$ & $\begin{array}{c}6.97 \\
\mathrm{~g} \\
\end{array}$ & \multirow{4}{*}{$\begin{array}{c}8.28 \\
\text { b }\end{array}$} \\
\hline & 500 & $\begin{array}{c}5.50 \\
\mathrm{p}\end{array}$ & $\begin{array}{c}7.70 \\
\text { no }\end{array}$ & $\begin{array}{c}7.70 \\
\text { no }\end{array}$ & $\begin{array}{c}7.17 \\
0\end{array}$ & $\begin{array}{c}7.02 \\
\mathrm{~g}\end{array}$ & \\
\hline & 1000 & $\begin{array}{c}7.90 \\
\text { no }\end{array}$ & $\begin{array}{c}9.07 \\
\mathrm{~m} \\
\end{array}$ & $\begin{array}{c}8.77 \\
\mathrm{mn} \\
\end{array}$ & $\begin{array}{c}7.13 \\
\mathrm{o} \\
\end{array}$ & $\begin{array}{c}8.22 \\
\mathrm{f} \\
\end{array}$ & \\
\hline & 2000 & $\begin{array}{c}12.07 \\
\mathrm{j}\end{array}$ & $\begin{array}{c}11.47 \\
\mathrm{jk}\end{array}$ & $\begin{array}{c}11.00 \\
\mathrm{j}-1\end{array}$ & $\begin{array}{c}9.20 \\
\mathrm{~m}\end{array}$ & $\begin{array}{c}10.93 \\
\mathrm{e} \\
\end{array}$ & \\
\hline \multicolumn{2}{|c|}{ humic effect } & $\begin{array}{c}11.98 \\
\mathrm{c} \\
\end{array}$ & $\begin{array}{c}14.75 \\
\mathrm{a}\end{array}$ & $\begin{array}{c}13.83 \\
b\end{array}$ & $\begin{array}{c}11.63 \\
\mathrm{c}\end{array}$ & \multirow{3}{*}{$\begin{array}{c}\text { Ascorbic acid } \\
\text { effect }\end{array}$} & \\
\hline \multirow{2}{*}{ 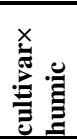 } & Khithairy & $\begin{array}{c}16.88 \\
\mathrm{c} \\
\end{array}$ & $\begin{array}{c}19.89 \\
\mathrm{a} \\
\end{array}$ & $\begin{array}{c}18.52 \\
\mathrm{~b}\end{array}$ & $\begin{array}{c}15.98 \\
\mathrm{~d}\end{array}$ & & \\
\hline & Sorany & $\begin{array}{c}7.08 \\
\mathrm{f}\end{array}$ & $\begin{array}{c}9.62 \\
\mathrm{e}\end{array}$ & $\begin{array}{c}9.15 \\
\mathrm{e}\end{array}$ & $\begin{array}{c}7.28 \\
\mathrm{f}\end{array}$ & & \\
\hline \multirow{4}{*}{ 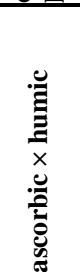 } & 0 & $\begin{array}{c}6.77 \\
\mathrm{j}\end{array}$ & $\begin{array}{c}15.22 \\
\mathrm{c}\end{array}$ & $\begin{array}{c}13.63 \\
\text { e3 }\end{array}$ & $\begin{array}{c}9.60 \\
\mathrm{i}\end{array}$ & $\begin{array}{c}11.30 \\
\mathrm{~d}\end{array}$ & \\
\hline & 500 & $\begin{array}{c}10.88 \\
\mathrm{~h}\end{array}$ & $\begin{array}{c}13.21 \\
\text { ef }\end{array}$ & $\begin{array}{c}12.67 \\
\mathrm{fg}\end{array}$ & $\begin{array}{c}11.23 \\
\mathrm{~h}\end{array}$ & $\begin{array}{c}12.00 \\
\mathrm{c}\end{array}$ & \\
\hline & 1000 & $\begin{array}{c}13.67 \\
\mathrm{e}\end{array}$ & $\begin{array}{c}14.48 \\
\mathrm{~d}\end{array}$ & $\begin{array}{c}13.60 \\
\mathrm{e}\end{array}$ & $\begin{array}{c}12.08 \\
\mathrm{~g}\end{array}$ & $\begin{array}{c}13.46 \\
b\end{array}$ & \\
\hline & 2000 & $\begin{array}{c}16.62 \\
\mathrm{a}\end{array}$ & $\begin{array}{c}16.10 \\
\mathrm{ab}\end{array}$ & $\begin{array}{c}15.43 \\
\text { bc }\end{array}$ & $\begin{array}{c}13.60 \\
\mathrm{e}\end{array}$ & $\begin{array}{c}15.44 \\
\mathrm{a} \\
\end{array}$ & \\
\hline
\end{tabular}

Means within a column, row and their interactions followed with the same letters are not significantly different from each other's according to Duncan's multiple ranges test at $5 \%$ level.

Khithairy cultivar had significantly more leaves fresh weight than Sorany cultivar. Ascorbic acid $\mathrm{x}$ humic acid interaction significantly influenced of leaves fresh weight, the highest value (16.62) was recorded in transplants that treated by only $2000 \mathrm{mg} . \mathrm{l}^{-1}$ ascorbic acid, and the lowest value(6.76) was recorded untreated transplants. Cultivar $\mathrm{x}$ humic acid interaction also significantly influenced leaves fresh weight, the highest value (19.89) was recorded in Khithairy transplants when treated with $20 \mathrm{mg} \cdot 1^{-1}$ humic acid, and the lowest value (7.08) was recorded in untreated Sorany cultivar. 
The interaction between cultivar and ascorbic acid notice that the Khithairy cultivar when treated with 2000mg. $\mathrm{l}^{-1}$ ascorbic acid gave the highest value (19.84), and the lowest value was recorded in untreated Sorany cultivar (6.97). Regarding cultivar $\mathrm{x}$ ascorbic acid $\mathrm{x}$ humic acid interaction significantly difference also noticed that Khithairy transplants treated with only $2000 \mathrm{mg} .1^{-1}$ ascorbic acid had significant differences compared with other interaction (21.17), and the lowest value (2.87) recorded in untreated Sorany cultivar.

\subsection{Leaves dry weight}

Results in table (10) notice the humic acid levels had significant influence on leaves dry weight when compared with the control.

Table (10) Effect of humic, ascorbic acid, cultivar and their interactions on leaves dry weight of olive transplant cvs. 'Khithairy ' and 'Sorany'.

\begin{tabular}{|c|c|c|c|c|c|c|c|}
\hline \multirow[b]{2}{*}{ 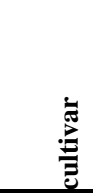 } & \multirow{2}{*}{ 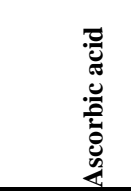 } & \multicolumn{4}{|c|}{ Humic concentration } & \multirow{2}{*}{$\begin{array}{l}\text { cultivar } \times \text { ascorbic } \\
\text { acid }\end{array}$} & \multirow{2}{*}{$\begin{array}{l}\text { Cultivar } \\
\text { effect }\end{array}$} \\
\hline & & $\mathbf{0}$ & 20 & 40 & 60 & & \\
\hline \multirow[b]{4}{*}{ 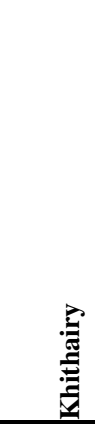 } & $\mathbf{0}$ & $\begin{array}{l}5.70 \\
\text { f }\end{array}$ & $\begin{array}{l}9.87 \\
\mathbf{a}\end{array}$ & $\begin{array}{l}8.97 \\
\text { ab }\end{array}$ & $\begin{array}{l}7.70 \\
\text { b-e }\end{array}$ & $\begin{array}{l}8.06 \\
b\end{array}$ & \multirow{4}{*}{$\begin{array}{l}8.24 \\
\text { a }\end{array}$} \\
\hline & 500 & $\begin{array}{l}7.03 \\
\text { e }\end{array}$ & $\begin{array}{l}8.63 \\
\text { ab }\end{array}$ & $\begin{array}{l}8.40 \\
\text { b-d }\end{array}$ & $\begin{array}{l}7.93 \\
\text { b-e }\end{array}$ & $\begin{array}{l}8.00 \\
b\end{array}$ & \\
\hline & 1000 & $\begin{array}{l}8.47 \\
\text { bc }\end{array}$ & $\begin{array}{l}8.97 \\
\text { ab }\end{array}$ & $\begin{array}{l}8.87 \\
\text { ab }\end{array}$ & $\begin{array}{l}8.80 \\
\text { ab }\end{array}$ & $\begin{array}{l}8.78 \\
a\end{array}$ & \\
\hline & 2000 & $\begin{array}{l}7.20 \\
\text { de }\end{array}$ & $\begin{array}{l}8.80 \\
\text { ab }\end{array}$ & $\begin{array}{l}8.37 \\
\text { b-d }\end{array}$ & $\begin{array}{l}8.20 \\
\text { b-e }\end{array}$ & $\begin{array}{l}8.14 \\
\text { b }\end{array}$ & \\
\hline \multirow[b]{4}{*}{ 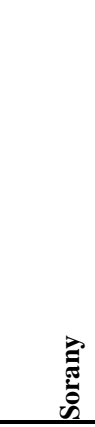 } & $\mathbf{0}$ & $\begin{array}{l}1.50 \\
\mathrm{~m}\end{array}$ & $\begin{array}{l}\mathbf{5 . 4 7} \\
\text { fg }\end{array}$ & $\begin{array}{l}4.80 \\
\text { g-j }\end{array}$ & $\begin{array}{l}2.90 \\
1\end{array}$ & $\begin{array}{l}3.67 \\
d\end{array}$ & \multirow{4}{*}{$\begin{array}{l}4.37 \\
\text { b }\end{array}$} \\
\hline & 500 & $\begin{array}{l}\text { 3.07 } \\
\text { kl }\end{array}$ & $\begin{array}{l}4.03 \\
\text { h-l } \\
\end{array}$ & $\begin{array}{l}4.97 \\
\text { f-h }\end{array}$ & $\begin{array}{l}3.40 \\
j-1\end{array}$ & $\begin{array}{l}3.87 \\
\text { d }\end{array}$ & \\
\hline & 1000 & $\begin{array}{l}3.67 \\
i-1\end{array}$ & $\begin{array}{l}4.33 \\
\text { g-j }\end{array}$ & $\begin{array}{l}3.90 \\
\text { h-l }\end{array}$ & $\begin{array}{l}4.23 \\
\mathrm{~g}-\mathrm{k}\end{array}$ & $\begin{array}{l}4.03 \\
d\end{array}$ & \\
\hline & 2000 & $\begin{array}{l}7.37 \\
\text { c-e }\end{array}$ & $\begin{array}{l}5.87 \\
\text { f }\end{array}$ & $\begin{array}{l}5.43 \\
\text { fg }\end{array}$ & $\begin{array}{l}5.00 \\
\text { f-h }\end{array}$ & $\begin{array}{l}5.92 \\
\text { c }\end{array}$ & \\
\hline \multicolumn{2}{|c|}{ Humic effect } & $\begin{array}{l}5.50 \\
\text { c }\end{array}$ & $\begin{array}{l}7.00 \\
\mathrm{a}\end{array}$ & $\begin{array}{l}6.71 \\
\mathrm{a}\end{array}$ & $\begin{array}{l}6.02 \\
b\end{array}$ & \multirow{3}{*}{ Ascorbic acid effect } & \\
\hline 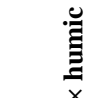 & Khithairy & $\begin{array}{l}7.10 \\
\text { c }\end{array}$ & $\begin{array}{l}9.07 \\
\mathrm{a}\end{array}$ & $\begin{array}{l}8.65 \\
\text { ab }\end{array}$ & $\begin{array}{l}8.16 \\
\text { b }\end{array}$ & & \\
\hline है & Sorany & $\begin{array}{l}3.90 \\
\text { e }\end{array}$ & $\begin{array}{l}4.93 \\
d\end{array}$ & $\begin{array}{l}4.78 \\
d\end{array}$ & $\begin{array}{l}3.88 \\
\mathrm{e}\end{array}$ & & \\
\hline \multirow{4}{*}{ 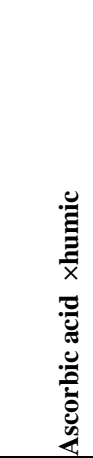 } & $\mathbf{0}$ & $\begin{array}{l}3.60 \\
\mathrm{~g}\end{array}$ & $\begin{array}{l}7.66 \\
a\end{array}$ & $\begin{array}{l}6.88 \\
\text { a-c }\end{array}$ & $\begin{array}{l}5.30 \\
\text { ef }\end{array}$ & $\begin{array}{l}5.86 \\
\text { c }\end{array}$ & \\
\hline & 500 & $\begin{array}{l}5.05 \\
\text { f }\end{array}$ & $\begin{array}{l}6.33 \\
\text { cd }\end{array}$ & $\begin{array}{l}6.68 \\
\text { bc }\end{array}$ & $\begin{array}{l}5.66 \\
\text { d-f }\end{array}$ & $\begin{array}{l}5.93 \\
c\end{array}$ & \\
\hline & 1000 & $\begin{array}{l}6.07 \\
\text { c-e }\end{array}$ & $\begin{array}{l}6.65 \\
\text { bc }\end{array}$ & $\begin{array}{l}6.38 \\
\text { cd }\end{array}$ & $\begin{array}{l}6.51 \\
b-d\end{array}$ & $\begin{array}{l}6.40 \\
b\end{array}$ & \\
\hline & 2000 & $\begin{array}{l}7.28 \\
\text { ab }\end{array}$ & $\begin{array}{l}7.33 \\
\text { ab }\end{array}$ & $\begin{array}{l}6.90 \\
\text { a-c }\end{array}$ & $\begin{array}{l}6.60 \\
\text { bc }\end{array}$ & $\begin{array}{l}7.03 \\
\mathrm{a}\end{array}$ & \\
\hline
\end{tabular}




\section{Means within a column, row and their interactions followed with the same letters are not significantly different from each other's according to Duncan's multiple ranges test at $5 \%$ level.}

The olive transplants when treated with $20 \mathrm{mg} . \mathrm{l}^{-1}$ humic gave the highest value (7.0)g, and untreated olive transplants gave the lowest value of leaves dry weight (5.50)g.

Whereas treating transplants with $2000 \mathrm{mg} \cdot \mathrm{l}^{-1}$ ascorbic acid increased leaves dry weight per transplant significantly that gave the highest value (7.03) $\mathrm{g}$ and the lowest value (5.86)g was recorded in untreated transplants.

The interaction between cultivar $\times$ ascorbic acid notice that the highest value (8.78) recorded in Khithairy cultivar and the lowest value (3.67) was recorded in untreated Sorany cultivar. Leaves dry weight per transplant was significantly increased by ascorbic acid $\mathrm{x}$ humic acid interaction, the spraying olive transplants with $20 \mathrm{mg} .1^{-}$

${ }^{1}$ only produced the highest leaves dry weight $(7.67) \mathrm{g}$, whereas the untreated olive transplants showed the lowest leaves dry weight per transplant (3.6)g. Concerning the effect of interaction between cultivar and humic acid levels, the highest value (9.07)g was recorded in Khithairy transplant that treated with $20 \mathrm{mg} . \mathrm{l}^{-1}$ humic and the lowest value (3.88)g was recorded in Sorany cultivar that treated with $60 \mathrm{mg} . \mathrm{l}^{-1}$ humic acid. Regarding interaction between cultivar $\mathrm{x}$ ascorbic acid levels significant differences in leaves dry weight per transplant were notice, the Khithairy transplants when treated with $1000 \mathrm{mg} .1^{-1}$ ascorbic acid gave the highest value $(8.78) \mathrm{g}$, and the lowest value (3.67)g was recorded in untreated Sorany transplants .Interaction between cultivars $\mathrm{x}$ ascorbic acid $\mathrm{x}$ humic acid levels, significant differences in leaves dry weight were notice, the highest dry weight were notice when the Khithairy transplants treated with only humic acid in level $20 \mathrm{mg} . \mathrm{l}^{-1}$ that gave $(9.87) \mathrm{g}$, and the lowest value (1.50) noticed in untreated Sorany cultivar.

\section{Conclusions:}

From the results of this study, it can be concluded that foliar application of ascorbic acid and humic and the interaction s between them had appositive effect on vegetative growth of olive transplants and Khithairy transplants cultivar had vigorous vegetative growth compared to the Sorany cultivar transplants.

\section{Reference}

[1] Bartolucci, P. and B.R. Dhakal (1999). Olive Growing in Nepal, TCP/NEP/6713, Field Document-1.

[2] Abdul-Qader, S.M. (2012). Effect of cultivar, organic manure, urea spray and their interactions on vegetative growth flowering, quantitative and qualitative characteristics of olive(Olea europaea L.), Ph.D. Thesis, Faculty of Agriculture and Forestry, Duhok University, Kurdistan Iraq.

[3] Mahdi, F.T. (2007). Development of olive plantation, Popular Company of Horticulture and Forestry, Ministry of Agriculture, Iraq

[4] Schachtschable, S.(1979). Lehrobuch der B odenkunde, printed in Germany (F.R.G.) Fedinand enke, stuttgrt.

[5] Farag, S. G.(2006). Minimizing mineral fertilizer in grapevine farm to reduce the chemical residuals in grapes. M.sc Thesis, instituteof environmental studies and research gypt, Ain shams university, Pp:67.

[6] Hegazi, E.S., M. R. El-Sonbaty, M. A. Eissa, D.M. Ahmed and T. F. El-Sharony (2007). Effect of organic and Biofertilization on vegetative growth and flowering of picual olive trees, world J. of Agric. Sci 3(2): 210-217.

[7] Nardi, S., g. Concheri and G. Dell'Agnola (1996). Biological activity of humus. In: Humic substances in terrestrial ecosystems (Piccolo A., ed). Elsevier, NY, USA. pp: 361-406.

[8] Chen, Y., M. De Nobili and T. Aviad (2004). Stimulatory effect of humic substances on plant growth. In Soil organic matter in sustainable agriculture. (Eds Magdoff F, Weil RR). Boca Raton, FL.

[9] Chen, Y, and T. Aviad (1990). Effects of humic substances on plant growth. In: McCarthy P, Calpp CE, Malcolm RL. Bloom, Readings. ASA and SSSA, Madison, WI. pp: 161-186.

[10] Varanini, Z.and R. Pinton (2001). Direct versus indirect effects of soil humic substances on plant growth and nutrition In The rhizosphere: biochemistry biochemistry and organic substances at the soil-plant interface (Pinton R Varanini Z., Nannipieri P., eds). Marcel Dekker Inc, NY, USA. pp: 141-157

[11] Casenave de Sanfilippo, E., J. A. Arguello,and G.A. Orioli .(1990)The effect of humic acids and their different molecular mass fractions on germination in sunflower Biolog. plantarum 32 42-48.

[12] Piccolo A, S. Nardi and G. Concheri (1992). Structural characteristics of humic substances as regulated to nitrate uptake and growth regulation in plant systems. Soil Biochem. 24: 373-380.

[13] Pizzeghello, D., G. Nicolini and S. Nardi (2002). Hormone-like activities of humic substances in different forest ecosystems. New Phytol., 155: 393-402.

[14] Gomez, M. L. and F.M. Lajolo (2008). Ascorbic acid metabolism in fruits: activity of enzymes involved in synthesis and degradation during ripening in mango and guava. J. Sci. Food and Agric. 88:756-762..

[15] Ahmed, F.F., A.M. Akl, A.A. Gobara and A.E. Mansour (1997). Yield and quality of Anna apple trees (Malus domestica) in response to foliar application of ascorbine and citrine fertilizer. Eygpt. J. Hort. 25(2): 120-139.

[16] Barth, C.; M.D. Tullio and P.L. Conklin (2006). The role of ascorbic acid in the control of flowering time and the onset of senescence. J. Experimental Botany, 57(8):1657-1665.

[17] Elham, D. Z. A. and M. F. M. Shahin (2006). Effect of spraying magnesium, boron, ascorbic acid and vitamin B complex on yield and fruit quality of "Canino" apricot. Arab Univ. J. Agric. Sci., Ain Shams Univ., Cairo. 14(1): 337-347.

[18] Maksoud, M. A., M.A. Saleh, M.S. El-Shamma and A. A. Fouad (2009). The beneficial effect of biofertilizers and antioxidants on olive trees under calcareous soil conditions. World J. Agric. Sci. 5 (3): 350-352.

[19] Smirnoff, N. and G.L. Wheeler, (2000). Ascorbic Acid in Plant. Critical Reviews in Plant Sciences, 19(4): 267-290.

[20] Hopkins, G.W.,( 1995). Introduction to Plant Physiology. pp 326-327. John Wiley and Sons, Inc. New York.

[21] Arrigoni, O.and M.C. De Tullio, (2000). The role of ascorbic acid in cell metabolism: between gene-directed functions and unpredictable chemical reactions. J. Plant Physiol., 157: 481-488. 
[22] Conklin, P.L. and C. Barth, (2004). Ascorbic acid, a familiar small molecule intertwined in the response of plants to ozone, pathogens, and the onset of senescence. Plant, Cell and Environ., 27: 959-971.

[23] H. Restrepo-Diaz, M. Benlloch, and R. Fernandez-Escobar,(2009). Leaf potassium accumulation in olive plants related to nutritional $\mathrm{K}$ status, leaf age, and foliar application of potassium salts, Journal of Plant Nutrition 32 .1108-1121.

[24] Agha, J.T. and D.A. Daoud,(1991). Evergreen fruit production, Part1. Mousl. Univ. Iraq, pp. 567-630. (in Arabic)

[25] Al-Rawi, K.M. and A. Khalafalla,(1980). Analysis of Experimental Agriculture Disgen, Dar Al-Kutub for Printing and Publishing, Mosul. University

[26] Statistical Analysis System, (2000)SAS Institute Inc., Cary, NC. USA.

[27] Rosado, R., M.C. Del Campillo, M.A. Martinez, V. Barron, and J. Torrent, (2002). Long-term effectiveness of vivianite in reducing iron chlorosis in olive trees, Plant and Soil 241; 139-144.

[28] Gobara, A.A. (1998). Response of Le-Cont pear trees of foliar application of some nutrients, Egypt. J. Hort. 25 ; 55-70. 